\title{
Availability growth and state-of-knowledge uncertainty simulation for offshore wind farms
}

\author{
A. Zitrou, T. Bedford, L. Walls \& K.Wilson \\ Department of Management Science \\ University of Strathclyde, Glasgow, UK \\ K. Bell \\ Department of Electronic \& Electrical Engineering \\ University of Strathclyde, Glasgow, UK
}

\begin{abstract}
The UK offshore wind sector has developed rapidly, but needs to develop even further to meet the low carbon targets set by the government. In order to increase capacity and reduce costs, new offshore wind farms will use larger and more complex technology that goes beyond current understanding and experience. As a result, predicting the technical performance of offshore wind farms involves a considerable amount of uncertainty, especially during early life when teething problems may arise. We present a model that allows us to distinguish between natural variability and state-of-knowledge uncertainty when assessing the growth in technical performance of offshore wind farms over early operational life. As such, the model can be used to inform decisions to reduce uncertainties and to support effective risk management. To describe wind farm performance, we use an indicator we call availability-informed capability measures the capacity of the farm to generate electricity. We use an example based on a two-phase Monte Carlo simulation to illustrate the use of the model.
\end{abstract}

\section{INTRODUCTION}

Offshore wind has a leading role in the future UK energy portfolio. Supported by the government, it has attracted considerable investment in recent years and has seen rapid growth. Nonetheless, in order to meet the government's ambitious low carbon targets, offshore wind capacity needs to grow even more. Building larger farms further away from shore will lead to better exploitation of the wind resource and increase capacity. However, this requires the development of novel large-scale technology. This fact introduces considerable technical risk and results in uncertain costs, making investment in offshore wind appear more risky. As such, the development of offshore wind can be encouraged by an improved understanding of the state of knowledge risks in the performance of offshore wind farms. There is a target of 97\% availability, used in the financial agreements on wind farms, which is a baseline for profitable performance.

Modelling the performance of an offshore wind farm of a relatively immature design over early life is particularly important, because, typically, this is a period of growth. Immature systems will typically under-perform with respect to prior predictions of long-term expected performance. In the case of largescale offshore wind farms, a number of design, manufacturing and operating issues could make system reliability lower than expected, while harsh weather along with organisational issues can increase system downtime. As operating experience accumulates and manufacturers and operators understand and address particular technical and operational issues, performance improves.

In this paper we present a mathematical model to represent uncertainties in offshore wind farm performance growth during early life. The model can be used to support decisions to reduce uncertainties and grow performance efficiently and effectively. The model is a simulation-based model developed in MATLAB. In this paper we present a simplified version of the model, but its structure allows the represention of more complex situations.

This paper is structured as follows: Section 2 discusses issues relevant to the modelling of offshore wind farm performance. In this section we distinguish between aleatory and state-of-knowledge uncertainty and we discuss the need to represent the latter in the modelling of offshore wind farms. We discuss the cur- 
rent wind farm modelling approaches and their limitations, and we introduce an availability-related performance measure that links technical performance with energy production. Section 3 presents the conceptual framework of the wind farm model disussed here and the basic mathematical structure for modelling reliability. A numerical example is used to illustrate the usefulness of the model. Finally, Section 4 concludes the paper.

\section{RAMS MODELLING OF OFFSHORE WIND FARMS}

\subsection{Modelling of uncertainties}

Modelling the technical performance of offshore wind farms involves considerable uncertainty. The stochastic nature of the failure and restoration processes that define the farm's performance, the use of novel technology and the harsh environmental conditions are all sources of uncertainty. Generally, uncertainties can be categorised into aleatory and state-of-knowledge uncertainties. Aleatory uncertainties relate to the natural variability of the world. For example, different turbines have fail at different times, even though they may be of identical design. Aleatory uncertainty, by definition, will always exist, and the best one can do is to describe it appropriately. In the case of offshore wind farm modelling, one can use stochastic processes to represent the aleatory uncertainty around failure and repair behaviours of wind turbines. Stateof-knowledge uncertainties, on the other hand, relate to the limitations of our knowledge about the world. For example, the lack of operating experience relevant to the novel offshore wind technology affects our ability to model turbine behaviours. However, unlike aleatory uncertainties, state-of-knowledge uncertainties can be reduced as more information is accumulated - for example through better testing before deployment.

When modelling offshore wind farm performance, it is important to account for both aleatory and stateof-knowledge uncertainties. State-of-knowledge uncertainties, unlike natural variability, are systemic. This is because problems arising from state-ofknowledge uncertainties affects all turbines in a wind farm in a similar manner. The implication is that availability might be systematically higher or lower than is being assessed currently. Since systematic lower performance is associated with lower revenues, this systemic risk affects the attractiveness of investing in offshore wind. However, once well-understood, one can identify ways to actively reduce state-of-knowledge uncertainties them by accumulating additional information and use tools such as value of information to value investment in testing. In general, distinguishing between aleatory and state-of-knowledge uncertainties is important because it allows decision-makers to identify how much control they have over risk.

\subsection{Current O\&M models}

Within recent years, there has been considerable interest in the development of tools to model the Operation \& Maintenance (O\&M) of an offshore wind farm. TU Delft (Bussel 1997) has developed a Monte Carlo method to yield estimates of farm availability and of O\&M costs over a predetermined period. ECN Wind Energy have developed the O\&M ECN model, an analytical model to determine annual average costs and downtime of a wind farm (Rademakers, Braam, Zaaijer, \& van Bussel 2003). The model is implemented in MS Excel using the @ Risk add-in and supports comprehensive uncertainty analysis. ECN has further developed the Operations and Maintenance Cost Estimator (OMCE) (Rademakers, Braam, Obdam, \& Pieterman 2009), a simulationbased model developed in MATLAB designed to support logistics decisions during the post-warranty period of the wind farm. Garrad Hassan and Partners have developed the O2M (Optimisation of Operations \& Maintenance) computer model (Phillips, Morgan, \& Jacquemin 2005) which simulates the operation of a wind farm to yield long-term average O\&M cost estimates, whereas the MWCOST model (Rowe, Dello, Frank, Brendling, \& Grittner 2000) is a simulation model developed initially for managing offshore oil and gas projects and further adopted for application in offshore wind contexts.

Current O\&M models typically represent failure behaviours of systems with mature reliability and assume constant rates for wind farm subassemblies. Moreover, to calculate wind farm downtime they typically consider aspects such as availability of maintenance equipment (e.g. hosting equipment), weather conditions (based on the analysis of wind and wave data) and duration of the required repair actions (assumed constant for different failure modes). Current O\&M models are large Monte Carlo simulations that are useful for optimising maintenance and controlling logistics that focus on modelling aleatory uncertainties only. The mathematical model presented here allows decision-makers to carry out state-of-knowledge analysis on the wind farm performance and it can be used to inform decisions to reduce risk of performance and grow capability more effectively and efficiently.

\subsection{Performance measure in terms of availability}

For offshore windfarms the most important measure of technical performance is farm availability. Indeed, the O\&M models discussed above yield estimates of average availability. But what does 'farm availability' really mean? There are two prevailing definitions of availability within the wind industry: turbine availability and system availability (Harman. 2008). Turbine availability describes the fraction of time that the turbine is able to produce energy, and it is the focus 
of warranties. System availability is the proportion of time that the entire farm delivers electricity to the grid, by considering all possible interruptions. Neither of these definitions are standardised, and the same availability term may correspond to different definitions (Feng, Tavner, \& Long 2010) (Robb 2010).

The model presented here yields estimates of a newly defined availability-related indicator that associates technical performance with energy production. This allows us to describe an individual turbine in terms of multiple operating states and define clearly the effect of degraded operation on overall farm performance (e.g. when a fault in the pitch system can lead to problematic alignment of the blades making the turbine unable to generate electricity at its full capacity). We call this indicator availability-informed capability.

Consider a wind farm comprising of $n$ wind turbines and that each turbine has $m+1$ operating states, where state $m$ is full technical performance and state 0 is out of operation. We use a stochastic process $\left\{X_{i}(t), t \geq 0\right\}$ with state space $\{0,1, \ldots, m\}$ (where $X_{i}(t)=j$ if turbine is in operating condition $j$ ) to model wind turbine $i$. We define the point availabilityinformed capability at time $t$ to be the fraction

$$
C(t)=\frac{\sum_{i=1}^{n} O P_{i}(t)}{\sum_{i=1}^{n} I P_{i}(t)}
$$

where $O P_{i}(t)$ is the maximum output power of turbine $i$ and $I P(t)$ is the turbine's rated output power (installed capacity). When the turbine is at a full operational state $(j=m)$ at time $t, O P_{i}(t)$ is equal to the installed capacity $I P_{j}(t)$, as it would provide full output if the wind were at full speed. The average farm availability-informed capability over some inter$\operatorname{val}\left(\tau_{1}, \tau_{2}\right)$ is given by

$$
C_{\left(\tau_{1}, \tau_{2}\right)}=\frac{1}{\tau_{2}-\tau_{1}} \int_{\tau_{1}}^{\tau_{2}} C(t) \mathrm{d} t
$$

We note that availability-informed capability describes the best performance that the farm could provide assuming that the wind were strong enough to drive the turbines at full speed. The reason we do not model energy output is that the objective of the model presented here is to inform decisions to reduce technical risk. An energy output measure would include epistemic and aleatory wind uncertainties hence compounding different kinds of uncertainty and making it more difficult to identify those key uncertainties around technical risks than could be subject to the control of the decision-maker.

SHIT

\subsection{Interventions to grow performance and reduce risk}

The performance of repairable systems, such as offshore wind turbines, depends on two factors: the failure behaviour of the system (reliability) and the restoration behaviour of the system (speed at which a system can go from an inoperable to an operable condition). To achieve performance growth, one can intervene and modulate either the turbine's failure behaviour, or its restoration behaviour, or both.

We consider three types of intervention:

Innovations These are radical actions that change the basic underlying properties of the system. For instance, design modifications can overcome design inadequacies while manufacturing faults can be removed by improving quality procedures.

Minor adjustments These are refinements of, for example, operational activities, that have a gradual impact on performance. For example, the learning effect of operational experience which could lower the probability of maintenance induced failures.

Maintenance strategy The maintenance strategy encompasses both the type of intervention (i.e. preventive or corrective) and the effect of intervention on the system condition (i.e. perfect or imperfect repair).

The focus of this paper is on the modelling of stateof-knowledge uncertainties on offshore wind farm availability performance, rather than the modelling of performance growth per se. In many cases, reducing uncertainty on performance predictions is equally important to improving performance. For details on the modelling of the effect of interventions on wind farm performance see (Zitrou, Bedford, \& Walls 2012).

It is worth mentioning that interventions may result not only in performance growth but also in risk reduction. Consider the design process of an offshore wind turbine. During the conceptualisation phase aspects of the operating environment are determined to assess the stresses that will affect reliability. Issues are identified and prioritised, to ensure that they are addressed by the design. Some of these issues are characterised as low risk, and a conscious decision is made not to address them during the design phase. When a 'low-risk' issue is in fact prevalent, or when a real issue is omitted, the system is susceptible to out-ofspecification stresses, leading to under-performance. This is one of the sources of state-of-knowledge uncertainty for immature systems.

Investing in a thorough testing process can ensure that all critical stresses are anticipated, allowing us not only to lower, but also to better predict the risk of overstress failures for subsequent versions of the 


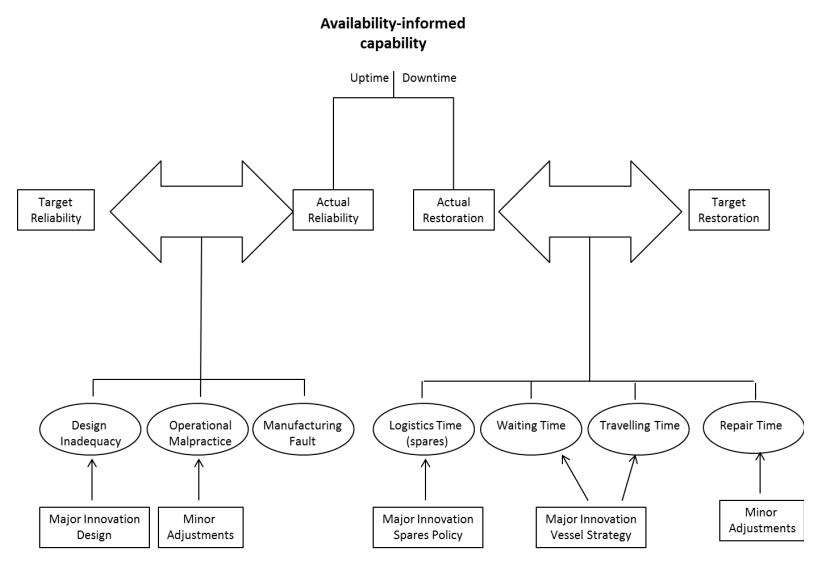

Figure 1: Conceptual model of failure process for a turbine

design. Similarly, longer testing intervals allow designers to gain better insight into e.g. damage accumulation mechanisms. Appropriate defences can then be built into the design, 'delaying' the wear-out process. At the same time, increased understanding of the physical processes allows for better reliability predictions.

\section{SIMULATION MODEL FOR AVAILABILITY GROWTH}

\subsection{Conceptual framework}

The model presented in this paper is a Monte Carlo simulation model developed in MATLAB. The model represents uncertainties on the projections of availability-informed capability over the early life of an offshore wind farm. Within this framework, the reliability of systems (wind turbines) comprising the farm are described in terms of non-decreasing rates. Restoration behaviours are determined in terms of aspects such as ease of repair, ease of access to the offshore site, availability of maintenance resources like vessels etc. The model represents the initial discrepancy between achieved and target performance due to design, manufacturing or operating errors and demonstrates how the former grows to meet the latter through interventions (see Figure 1).

As described in Section 2.3, farm capability describes the best performance that the farm could provide assuming that the wind were strong enough to drive the turbines at full speed. Wind resource is not modelled explicitly, but the effect of weater is captured through the waiting time (amount of time the crew waits until the weather is suitable for maintenance operations). This feature of the model allows us to consider the effect of weather on downtime without compounding weather uncertainty with state-ofknowledge uncertainty.

\subsection{Mathematical foundations}

Let $T_{1}, T_{2}, \ldots$ denote the successive times of events for a turbine which began operation at time $T_{0}=0$. To describe the alternating uptime-downtime behaviour of the repairable subassemblies of a turbine we use a marked point process. $\left\{T_{n}, J_{n}\right\}_{n \geq 1}$ where $J_{n}=1$ when a failure occurs at $T_{n}$ and $J_{n}=0$ otherwise $(n=$ $0,1,2, \ldots)$. Let $N(t)$ be the number of failures and $M(t)$ the number of restorations in $(0, t]$. The Rate of Occurrence of Failures (ROCOF) of the process is defined conditionally as

$$
\lambda(t)=\lim _{\Delta t \rightarrow 0} \frac{\operatorname{Pr}\left(N(t+\Delta t)-N(t) \mid \mathcal{H}_{t}\right)}{\Delta t}
$$

where $\mathcal{H}_{t}$ is the subassembly history, that is, a collection of everything we know about the subassembly until, but not including, time $t$.

To describe the ROCOF we combine two concepts: the inherent reliability characteristics of the turbine, as described by the Force of Mortality (FOM) $h(t)$, and the effect of maintenance represented by the virtual age of the turbine, $v(t)$. The FOM describes the first time to failure of the turbine and virtual age represents its condition at some point in time. We have

$\lambda(t)=h(v(t)), \quad t>0$.

For a new system we have $v(t)=0$. Therefore, perfect maintenance 'resets' the virtual age of the turbine to zero, whereas minimal repair sets its value to the one it had just before failure.

Our model for failure behaviour is deliberately simple with a small number of parameters. It assumes an early phase in which there is a constant force of mortality, which ends when aging begins which follows a Weibull shape. The parametric form of the FOM is given by

$h(t)= \begin{cases}\lambda & , t \leq s \\ \lambda+a b(t-s)^{b-1} & , t>s\end{cases}$

where $\lambda$ is the FOM of the turbine under random failures and $a>0$ and $b>1$ are the scale and shape parameters respectively of the non-decreasing FOM characterising the turbine's wear-out phase. Time $s$ is the point when the turbine moves from constant to a non-decreasing FOM.

For a turbine whose failure characteristics are as planned, the rate $\lambda$ will be low, the time $s$ outside the early life window (Figure 2(a)), and the rate of increase of the FOM will be slow. However, when the design is novel there is a risk that design, production and operation errors will cause the system to initially under-perform. As illustrated in Figure 2(b), random failures may be more frequent $\left(\lambda^{\prime}>\lambda\right)$ and/or wearout may start prematurely $\left(s^{\prime}<s\right)$ during early life. Modelling the failure behaviour of an offshore wind turbine involves considerable state-of-knowledge uncertainty, including a non-zero probability that there are substantial design and manufacturing issues. We capture this uncertainty by introducing a set of possible FOMs, one of which is the planned FOM shown in Figure 2(a), and selecting the initial FOM for all turbines randomly from this set. 
As state-of-knowledge uncertainty is knowledgebased, it is possible to reduce it by learning more about the model parameters. For instance, collecting more information about the operating environment and the stresses that this imposes on a turbine improves the estimation of the scale parameter $a$. A better insight into e.g. the degradation failure mechanisms leads to better predictions of time $s$. Collection and analysis of early operation data or a more thorough design phase provides insight into parameter $p$. Major interventions and minor adaptations influence some of the parameters of the FOM (e.g. a design update may change the shape parameter $a$ or/and the 'phase-switching' parameter $s$ ), whereas the learning effect will impact on probability $p$ (as operating experience accumulates, the chance of e.g. operating error decreases).

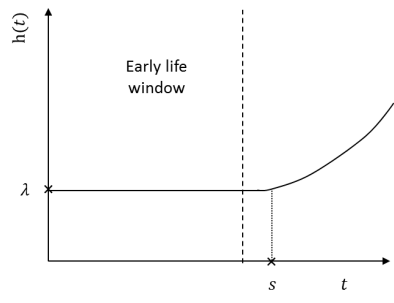

(a) Target

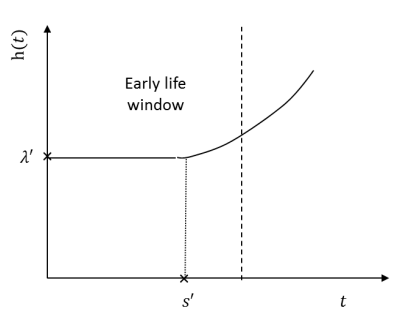

(b) Below target
Figure 2: Force of Mortality (FOM) of a wind turbine

The model presented in this paper uses Monte Carlo simulation to propagate the state-of-knowledge uncertainties in the turbine's FOM's to assess the overall risk in availability performance growth of offshore wind farms during early operational life. This model can provide insight into the effectiveness of interventions and will inform management decisions to buy down uncertainties and grow availability more effectively and efficiently.

\subsection{Illustrative example}

In this section we illustrate the use of the MATLAB model to represent uncertainty in early-life performance assessments of an offshore wind farm by using a simple example. To distinguish between state-ofknowledge and aleatory uncertainties, we use a Monte Carlo approach with two nested 'loops': in the first loop we consider the state-of-knowledge uncertainty and in the second, nested, loop we consider aleatory uncertainty. This way, we are able gain better insight into the scale and source of uncertainties and inform decisions to manage them appropriately.

Consider a farm comprising of $N=100$ identical turbines. Suppose that this is a large-scale farm at a pre-construction stage, comprising of 5MW turbines. The interest lies in predicting the performance of the farm over its early life, assumed to cover the first five years of operation. Early life typically coincides with the warranty period. As this is an illustrative example, we are making some simplifying assumptions. We assume that each turbine can be in one out of two operating states (functioning or non-functioning). Turbines are subject to corrective maintenance, and after repair, they are returned to the condition they were just before failure (minimal repair). Moreover, yearly overhauls restore the turbines to an as good as new condition. Overhauls are staggered, as it is not practical to take all turbines out of operation at the same time. Finally, design updates are fitted to the turbines during the summer months of years 2 and 3, whereas manufacturing errors are removed during the summer months of year 2 (innovations).

We note that the model can be easily modified to capture more complex situations. For instance, if required, each turbine can be broken down to subsystems (subassemblies like the gearbox or the yaw system). Moreover, the use of availability-informed capability as a performance measure as defined in Section 2.3 allows us to extend the number of states and capture partial turbine performance. Finally, the use of the virtual age concepts allows as to model other types of imperfect maintenance.

The turbines comprising the farm are assumed large-scale machines of novel design. The turbine manufacturer anticipates a reliability level of a ROCOF of $\lambda=0.1$, or, equivalently, a Mean Time To Failure (MTTF) equal to 10 years. We assume that the average restoration duration of a failed turbine is equal to 0.25 years. The stated reliability level can be expected if there are no teething problems with the design, manufacturing or operation of the turbine. Nevertheless, scaling up to such a large machine size implies a large amount of innovation, introducing significant risk. For instance, increased rotor diameters impose high load levels on the turbine structure (e.g. blades, tower, gearbox and generator).

To withstand the higher loads, the material properties have to be chosen correctly. If proper material is used then the target reliability is achieved; however, a design inadequacy such as the use of inappropriate material can occur with a $20 \%$ chance $\left(p_{1}=0.2\right)$ and lead to premature wear-out. The state-of-knowledge uncertainty on the wear-out mechanism given a design inadequacy is expressed with a probability distribution on the scale parameter $a$ of the FOM given in (5) (see Figure 3). In addition, the change in the scale introduces a number of fresh manufacturing challenges. Assume that there is a 0.1 chance that a new turbine has a manufacturing flaw $\left(p_{2}=0.1\right)$. In case of a defect the turbine matures prematurely.

Our model is based on a two-stage Monte Carlo simulation. In the first stage we have a loop with $n_{1}=100$ iterations (first loop), for the purposes of which we generate:

- $n_{1}$ variables from the Bernoulli distribution with probability $p_{1}=0.2$, where $X_{1, j}=1(j=$ $\left.1, \ldots, n_{1}\right)$ if the material used is inappropriate 


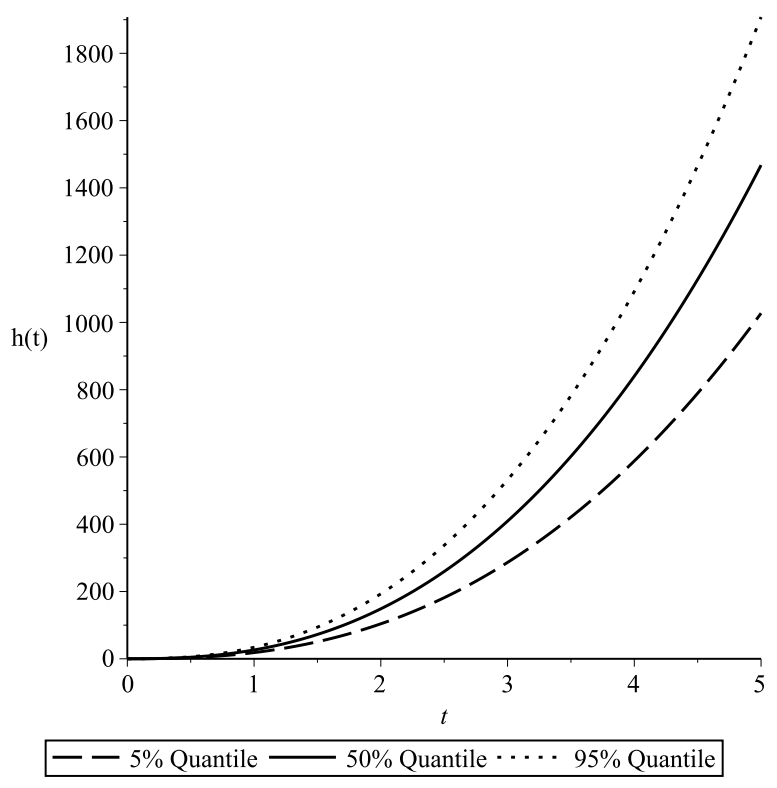

Figure 3: FOM curves given a design inadequacy (inappropriate material) corresponding to different values of the scale parameter $a$.

- $n_{1} \times N$ variables from the Bernoulli distribution with probability $p_{2}=0.1$, where $X_{2,(i, j)}=1$ $\left(i=1, \ldots, N\right.$ and $\left.j=1, \ldots, n_{1}\right)$ if turbine $i$ in iteration $j$ has a manufacturing fault

- $n_{1}$ variables from the uniform distribution $U(5,10)$, where $a_{j}$ is the scale parameter of the FOM given a design inadequacy.

For each first-stage iteration, parameter vector

$$
\left[X_{1, j}, X_{2,(1, j)}, \ldots, X_{2,(N, j)}, a_{j}\right]
$$

determines a single curve for the turbine FOM. In the second stage of the simulation, we have a nested loop with $n_{2}=100$ iterations (second loop) for each first-loop iteration. For each second-loop iteration we calculate a probability distribution for the wind farm time-averaged availability-informed capability, which represents aleatory uncertainty only. Figure 4 gives an example of a single empirical cumulative distribution curve representing this aleatory uncertainty.

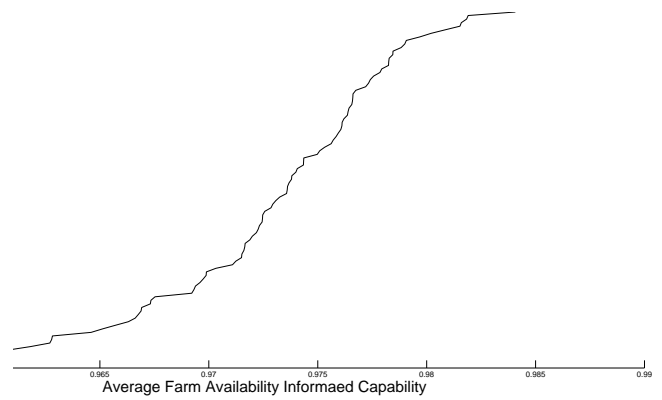

Figure 4: A single run of loop one of the simulation representing the state of knowledge uncertainty on the average availability informaed capability of the farm.

The figure shows that, for this particular second loop iteration, the farm is almost certain to have an average availability informed capability of at least $96 \%$ but will reach $97.5 \%$ with a probability of less than a half.

Figure 5 portrays the collection of the $n_{1}$ distributions obtained from running the first-loop simulation. The spread of the distributions represents state-ofknowledge uncertainty. The vertical dotted line represents an acceptable level of capability, in this case equal to $97 \%$. The distribution of the empirical cumulative probabilities of each of these curves along the vertical line determines the probability that this level of performance will be achieved during the early life of the farm.

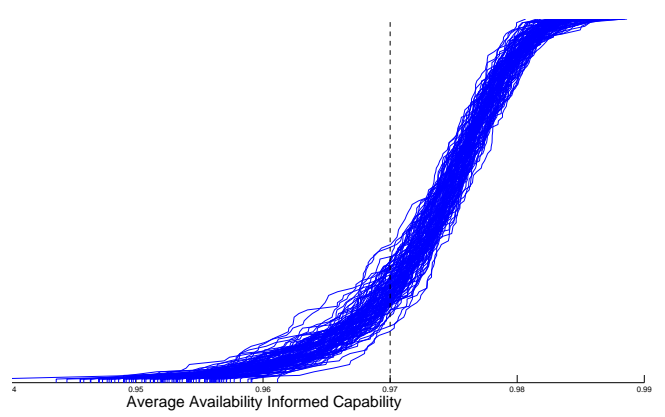

Figure 5: Multiple runs of loop one of the simulation representing the state of knowledge uncertainty on the average availability informaed capability of the farm.

From the figure we see that the greatest state-ofknowledge uncertainty around the average availability informed capability is towards the central portion of the empirical cumulative distribution function. This is also close to our target performance level for the farm.

We can take a vertical slice through the curves at our dotted line to plot the distribution of the empirical cumulative probabilities at the target level. This shows the state-of-knowledge uncertainty, around the probability of hitting the $97 \%$ target.

This histogram is given in Figure 6.

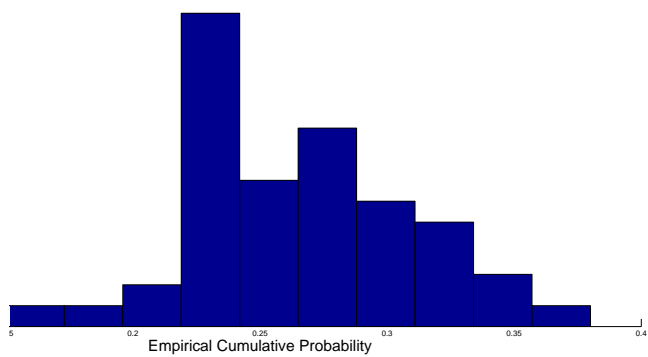

Figure 6: A histogram representing the empirical probabilities of not reaching the farm target capability in each of the simulations.

From the histogram we see that the probability that the farm fails to make its target average availability informed capability of $97 \%$ is between 0.2 and 0.4 . It is most likely to be between 0.25 and 0.3 . 
Now, suppose that extensive laboratory testing can increase the likelihood that new turbine designs can provide planned reliability levels. Investing in more comprehensive testing before the turbine is deployed can decrease probability $p$ to 0.05 . It is of interest to see how additional testing impacts on availability performance risk. Figure 7 represents the results of the two-stage simulation.

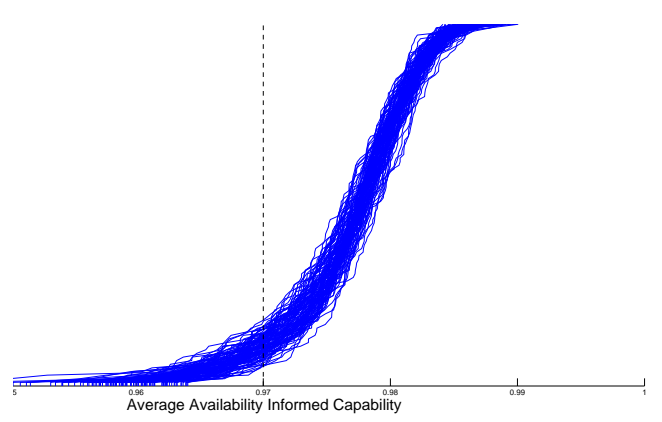

Figure 7: A plot of the epistemic uncertainty resulting from simulations with $p$ reduced to 0.05 .

It can be seen that the spread of the distributions has been decreased, and investing in testing results in better descriptions of the scale of the risk to the company. It also has the added advantage of reducing the probability that the farm will fail to meet it's target performance.

We can compare the results using the two different values of $p$ more directly using a density plot. This is given in Figure 8.

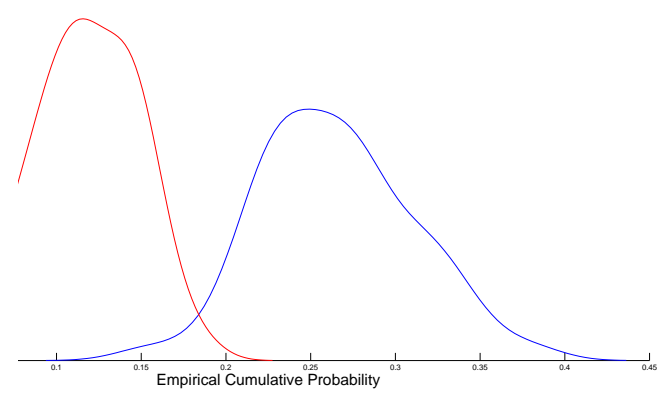

Figure 8: A plot of the epistemic uncertainty at the critical availability resulting from simulations with $p$ equal to 0.1 (right curve) and 0.05 (left curve).

We can see more clearly from this plot the two results we discussed for the previous plot. The density for the smaller value of $p$ has a smaller spread than that with larger $p$, indicating that the state-ofknowldege uncertainty has been reduced. This density has also been shifted to the left indicating that the probability of the farm failing to reach its target availability has also decreased. The fact that the densities hardly overlap indicates that this decrease is significant.

\section{CONCLUSION}

In this paper we presented a model for the representation of uncertainties on performance projection of an offshore wind farm, particularly during the early operational years. The model determines farm availability performance by modelling the reliability and restoration processes and captures the effect of interventions on the failure and restoration behaviours of the individual turbines. The focus of this paper is on the modelling of uncertainties on wind farm availability performance projections. This is particularly important for offshore wind farms, where the use of novel technology introduces a significant amount of performance risk.

To demonstrate the use of the presented model, we use a simple simulation example developed in MATLAB. Based on a two-stage Monte Carlo simulation, the model accounts for both aleatory and state-ofknowledge uncertainties, and distinguishes between the two in the context of assessing the performance of an offshore wind farm during early life. This is important as decision-makers can gain insight into the scales of different uncertainties and inform decisions to buy down uncertainties that are under their 'control', or potentially transfer risk to another party through e.g. insurance covers or contracts. In the example the model is used to explore how the scale of uncertainties change if one invests in more comprehensive testing.

The work presented in this paper is part of a larger model for assessing the availability performance growth of offshore wind farms during early life. The farm model will be used to understand the factors that drive early availability and the effect of modulating these, in order to inform decisions to grow availability efficiently and effectively and manage uncertainties and technical risk. The model will be comprised of a number of different FOM's that describe the multiple wind turbines sub-assemblies in the farm and allow for the modelling of more complex maintenance strategies, such as condition monitoring.

Future work involves eliciting expert judgement to gain insight into the uncertainties related to reliability issues relevant to offshore wind turbines. We envisage to develop a generic model for offshore wind farm availability performance growth, that can be customised to support decisions relevant to a particular case.

\section{REFERENCES}

Bussel, G. v. (1997). Operation and maintenance aspects for large offshore wind farms. In EWEC, Dublin, Ireland, pp. 272-275.

Feng, Y., P. Tavner, \& H. Long (2010). Early experiences with uk round i offshore wind farms. Energy 163(EN4), 167-181.

Harman., K. (2008). Availability trends observed at operating wind farms. In Proceedings of the European Wind Energy Conference. European Wind Energy Association. 
Phillips, J., C. Morgan, \& J. Jacquemin (2005). Understanding uncertainties in energy production estimates for offshore wind farms. In Copenhagen Offshore Wind 2005. Garrad Hassan and Partners, Bristol, UK.

Rademakers, L., H. Braam, T. Obdam, \& R. Pieterman (2009). Operation and maintenance cost estimator (OMCE) to estimate the future $\mathrm{O} \& \mathrm{M}$ costs of offshore wind farms. Technical Report ECN-M-09-126, ECN: Energy Research Institute in the Netherlands.

Rademakers, L., H. Braam, M. Zaaijer, \& G. van Bussel (2003). Assessment and optimisation of operation and maintenance of offshore wind turbines. In European Wind Energy Conference, Madrid, Spain.

Robb, D. (2010, December). Finding fault: Improving wind farm availability.

Rowe, S., S. Dello, J. Frank, W. Brendling, \& S. Grittner (2000). Simulating operating \& production efficiencies for deep water field developments. In Offshore Technology Conference, Houston, Texas.

Zitrou, A., T. Bedford, \& L. Walls (2012). Modelling the availability of offshore wind farm sub-assemblies. In 11th International Probabilistic Safety Assessment and Management Conference and the Annual European Safety and Reliability Conference 2012 (PSAM11 ESREL 2012), pp. 4741-4750.

\section{ACKNOWLEDGEMENTS}

This research was supported by EPSRC grant EP/I017380/1. We gratefully acknowledge support and contributions from the Department of Electronic and Electrical Engineering of University of Strathclyde. 